$>$ Qu'est-ce qu'une cellule souche? La propriété souche est-elle une propriété intrinsèque ou extrinsèque ? Quel rôle joue le microenvironnement tumoral dans l'identité de la propriété souche? $\varepsilon$ n fonction des réponses à ces questions, nous distinguons quatre identités pour les cellules souches normales et cancéreuses et explorons leur impact sur le choix de la stratégie thérapeutique en oncologie. L'acquisition d'altérations génétiques et épigénétiques au cours de la transformation maligne et de la progression remet en question la stabilité de l'identité de la propriété souche dans les cancers. <

\section{Identité des cellules souches normales et cancéreuses}

Lucie Laplane $^{1,2}$, Éric Solary ${ }^{2,3}$
La notion de cellule souche s'applique à une grande diversité de cellules qui ont en commun une position généalogique: ce sont des cellules à partir desquelles sont générées les cellules plus matures. Mais il existe différents types de cellules souches dans différents tissus, à différents stades du développement et dans différentes espèces. Ces cellules souches diffèrent dans leurs capacités fonctionnelles (leur potentiel de différenciation, par exemple), leurs propriétés moléculaires (les gènes qu'elles expriment) et leur phénotype (leurs marqueurs de surface). Cette diversité questionne l'unicité de l'identité souche. Quel type de propriété est la propriété souche ${ }^{1}$ ? À travers une analyse critique, portée par une philosophe des sciences et un médecin-chercheur en hématologie, nous montrerons que quatre identités souches peuvent être distinguées et examinerons les conséquences de chacune d'entre elles pour le traitement des cancers. En explorant plus spécifiquement le système hématopoïétique, nous interrogerons la stabilité de l'identité de la propriété souche en contexte pathologique.

Vignette (Photo (c) Inserm-Laboratoire de neurobiologie et transgenèse EA 3143). ${ }^{1}$ La traduction française du concept de stemness interroge souvent. Le choix du radical -ité (souchéité) indiquerait une caractéristique alors que le radical-itude (souchitude) indiquerait une abstraction. Toutefois, la dérivation par suffixe se faisant à partir des adjectifs et non des noms communs, il est plus légitime de traduire stemness par «propriété souche » ou « caractère souche».
${ }^{1}$ CNRS U8590 - Institut d'histoire et de philosophie des sciences

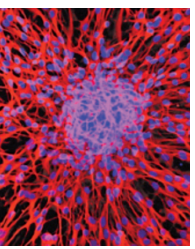

et des techniques (IHPST), université Paris I Panthéon-Sorbonne, 13, rue du Four, 75006 Paris, France ;

2Inserm U1170, Gustave Roussy, 114, rue Edouard Vaillant, 94800 Villejuif, France;

${ }^{3}$ Faculté de médecine Paris-Sud, 94270 Le Kremlin-Bicêtre, France. lucie.laplane@univ-parisl.fr

\section{La propriété souche peut-elle être acquise} par des cellules non-souches?

La conception classique selon laquelle la différenciation des cellules souches vers les cellules matures serait irréversible est remise en question. Le clonage et, plus récemment, la production des cellules souches pluripotentes induites ont montré que le destin des cellules matures n'est pas irrémédiablement scellé. In vitro, l'introduction de quelques facteurs de transcription suffit à reprogrammer une cellule, à modifier son identité. Mais qu'en est-il de l'identité cellulaire in vivo? $\varepsilon n$ biologie végétale, la plasticité cellulaire est admise [1]. La notion de dédifférenciation est également acceptée dans les espèces capables de régénération comme la salamandre. Elle est beaucoup plus discutée chez les mammifères, et singulièrement dans l'espèce humaine. Toutefois, l'idée que les cellules épithéliales sont capables de dédifférenciation et de plasticité, notamment en contexte de réparation, progresse rapidement [2-5] tout comme celle d'une dédifférenciation accrue associée à l'oncogenèse (discutée dans [6]) $(\rightarrow)$.

\section{$\rightarrow$ Voir la Synthèse de C. Moyret-Lalle et al., $m / s n^{\circ} 8-9$, août-septembe 2016, page 725}

\section{Quel rôle exerce le microenvironnement dans l'identité souche?}

Parce qu'elle fait partie d'un organisme pluricellulaire, chaque cellule souche est dans un microenvironnement, au contact d'autres cellules et de facteurs solubles, dont certains qu'elle synthétise et 
sécrète elle-même. Dès lors se pose la question de la contribution de ce microenvironnement à l'identité de la propriété souche.

La compréhension de la propriété souche peut-elle s'abstraire du microenvironnement? Dans le système hématopoïétique par exemple, la notion de cellule souche a d'abord été pensée indépendamment de son environnement, comme une fonction autonome définissant une catégorie de cellules capables de générer l'ensemble des cellules hématopoïétiques. En introduisant le concept de «niche» cellulaire pour désigner le microenvironnement des cellules souches hématopoïétiques et en lui attribuant un rôle déterminant dans le maintien et le contrôle de cette identité, Ray Schofield a introduit dans les années 1970 un tournant conceptuel qui s'est généralisé en biologie avec une attention croissante portée au rôle du microenvironnement dans tous les tissus [7, 8].

Si le microenvironnement joue un rôle déterminant dans l'identité souche, s'agit-il du contrôle de l'activité souche ou de l'induction de cette propriété souche dans des cellules non-souches? Dans le système hématopoïétique, la niche est conçue comme une entité de contrôle qui régule l'activité des cellules souches. Mais des travaux ont montré que la niche peut, dans d'autres systèmes, induire la propriété souche. Par exemple, chez la drosophile et la souris, les progéniteurs germinaux peuvent se dédifférencier en cellules souches germinales après transplantation, irradiation ou du fait du vieillissement, et cette dédifférenciation peut également être obtenue in vitro en présence de facteurs de croissance produits par la niche $[9,10]$.

\section{Quelles identités possibles pour la propriété souche?}

L'identité de la cellule souche est régulièrement discutée autour d'une alternative binaire : s'agit-il d'un «type » cellulaire ou d'un «état» cellulaire [11] ? d'une «entité »ou d'un «état» [12]? ( $\rightarrow$ ) d'une «entité » ou d'une «fonction» [13] ? d'une identité « innée » ou d'une identité « acquise » (nature $\rightarrow$ Voir la Synthèse de D. Zipori, $m / s n^{\circ} 3$, mars 2011, page 303 or nurture) [14]?

Derrière cette apparente binarité se cachent en fait deux questions distinctes: (1) Des cellules non-souches peuvent-elles acquérir la propriété souche? (2) Le microenvironnement joue-t-il un rôle déterminant dans l'identité de la propriété souche? De la réponse à ces questions résultent non pas deux mais quatre identités possibles pour la propriété souche: catégorique, dispositionnelle, relationnelle et systémique (Tableau I) (pour une analyse plus détaillée, voir [15]).

La propriété souche peut être intrinsèque et autonome.

Il s'agit d'une propriété catégorique (une propriété dont dispose une entité du fait de ses caractéristiques intrinsèques et non en vertu de ses interactions avec le monde). C'est ainsi que s'est construit le concept de cellule souche lors de l'émergence de la théorie cellulaire au $x_{X} x^{e}$ siècle et de son expansion au cours du $x x^{e}$ siècle.
La propriété souche peut être intrinsèque et modulée par le microenvironnement.

Il s'agit d'une propriété dispositionnelle (une propriété qui est intrinsèque mais dont l'expression dépend de facteurs extrinsèques). La conception la plus classique du rôle de la niche cellulaire, en particulier dans le système hématopoiétique, suggère cette identité pour la propriété souche.

\section{La propriété souche peut être extrinsèque,} déterminée par le microenvironnement.

Il s'agit d'une propriété relationnelle (une propriété qui émerge d'une relation entre deux entités). Cette situation de niche inductrice est illustrée par les cellules souches germinales de la drosophile et de la souris.

La propriété souche peut être maintenue et régulée à l'échelle de la population cellulaire indépendamment de son microenvironnement.

II s'agit d'une propriété systémique (une propriété régulée et déterminée par le système). Par exemple, une expérience conduite sur des lignées de cancers du sein montre que les cellules matures purifiées et cultivées in vitro retournent à l'équilibre au cours du temps en régénérant notamment une nouvelle population de cellules souches cancéreuses [16].

\section{Le cas des cellules souches cancéreuses}

Toute distinction conceptuelle soulève la question de sa pertinence scientifique. Quel gain y $a-t$-il à distinguer quatre identités plutôt que deux? A-t-on besoin d'une telle classification?

La théorie des cellules souches cancéreuses (CSC), dont I'histoire peut être retracée depuis l'émergence de la théorie cellulaire au milieu du $x_{1}{ }^{e}$ siècle, mais qui a véritablement pris son essor à la fin des années 1990, postule que la hiérarchie de la différenciation cellulaire est maintenue ou mimée dans les cancers [15]. Les cellules cancéreuses seraient générées, comme dans les tissus sains, par un nombre limité de cellules aux propriétés souches. La théorie des CSC se heurte à un certain nombre de difficultés, en particulier :

- L'origine des CSC est inconstante; elles ne proviennent pas nécessairement de la transformation de cellules souches normales.

- Leur nombre est variable; les CSC représentent une fraction plus ou moins importante de la population cellulaire d'une tumeur donnée.

- Leur plasticité diverge ; des cellules cancéreuses non souches peuvent dans certains cancers devenir des CSC [17]. 


\begin{tabular}{|c|c|c|c|}
\hline $\begin{array}{l}\text { Identité de la } \\
\text { propriété souche }\end{array}$ & Définition & Exemple & Illustration biologique \\
\hline Propriété catégorique & $\begin{array}{l}\text { Propriété dont dispose une entité du fait } \\
\text { de ses propriétés intrinsèques et non en } \\
\text { vertu de ses interactions avec le monde }\end{array}$ & Masse & $\begin{array}{l}\text { CSC dans les syndromes myéloproli- } \\
\text { fératifs? [29-31] }\end{array}$ \\
\hline Propriété dispositionnelle & $\begin{array}{l}\text { Propriété intrinsèque dont l'expression } \\
\text { dépend de facteurs extrinsèques }\end{array}$ & Fragilité & $\begin{array}{l}\text { Cellules souches hématopoïétiques? } \\
\text { [8] }\end{array}$ \\
\hline Propriété relationnelle & $\begin{array}{l}\text { Propriété qui émerge d'une relation entre } \\
\text { deux entités }\end{array}$ & Poids & $\begin{array}{l}\text { Cellules souches germinales? } \\
{[9,10]}\end{array}$ \\
\hline Propriété systémique & Propriété déterminée par le système & Ailier gauche (football) & $\begin{array}{l}\text { CSC dans des lignées de cancer } \\
\text { du sein? [16] }\end{array}$ \\
\hline
\end{tabular}

Tableau I. Définitions des concepts philosophiques. CSC : cellules souches cancéreuses (pour une analyse plus détaillée, voir [15]).

La théorie des CSC a conduit à proposer un changement de stratégie thérapeutique. Plutôt que de chercher à éliminer le plus grand nombre de cellules cancéreuses comme le font les traitements anti-cancéreux classiques (la « réponse au traitement » est généralement évaluée sur la réduction du volume tumoral ou du nombre de cellules leucémiques sans autre précision), il serait «nécessaire et suffisant» d'éliminer spécifiquement les CSC au sein d'une tumeur pour l'éradiquer (la réponse devrait alors être évaluée sur le nombre de CSC résiduelles) [18]. La remise en question de «l'oligarchie des CSC » [19] $(\rightarrow)$ ne réduit pas la pertinence des CSC comme cible thérapeutique, mais elle augmente le nombre de cellules à éliminer pour obtenir la guérison. La pertinence de cette stratégie théra-

\section{$(\rightarrow)$ Voir la Nouvelle} de S. Häfner et L. Coulombel, $m / s n^{\circ} 3$, mars 2009, page 227 peutique repose cependant sur un présupposé que nous avons montré problématique, à savoir que la propriété souche est une propriété intrinsèque aux CSC, qu'elle soit catégorique ou dispositionnelle. $\mathrm{Si}$ la propriété souche est une propriété relationnelle ou systémique, la capacité des cellules cancéreuses non souches à générer de nouvelles CSC entravera la guérison.

Une stratégie thérapeutique alternative consiste à cibler la niche des CSC. Elle repose sur le présupposé que le microenvironnement joue un rôle déterminant dans l'identité de la propriété souche, conçue alors comme une propriété dispositionnelle ou relationnelle. Certaines situations expérimentales soutiennent cette conception. Par exemple, dans un modèle murin de leucémie myéloïde chronique, la neutralisation d'une cytokine produite par les cellules matures du clone leucémique bloque la progression de la maladie, indiquant le rôle essentiel d'un facteur extrinsèque à la CSC dans sa capacité à développer la maladie [20, 21]. Si l'identité de la propriété souche n'est pas dispositionnelle ou relationnelle, le ciblage du microenvironnement perd sa pertinence. II aura peu d'effet sur les CSC si elles ont une identité catégorique et des cellules cancéreuses non souches pourront encore les remplacer en cas d'identité systémique.

$\varepsilon$ n résumé, en distinguant quatre identités pour la propriété souche, la pertinence des objectifs thérapeutiques en cancérologie varie. Les présupposés sur l'identité de la propriété souche des CSC conduisent à cibler soit les CSC proprement dites, soit leur niche. II ne s'agit pas nécessairement d'alternatives, il est possible que ces deux approches se complètent, et la situation varie probablement d'un cancer à un autre, d'un patient à un autre, voire chez un même patient, tant les tumeurs deviennent hétérogènes en progressant. Ces deux stratégies thérapeutiques devraient avoir une efficacité comparable en cas d'identité dispositionnelle, mais le ciblage des CSC sera plus efficace en cas d'identité catégorique alors que celui de la niche sera plus efficace en cas d'identité relationnelle. Aucune de ces deux stratégies thérapeutiques ne s'applique efficacement à l'éradication des CSC en cas d'identité systémique, nous redirigeant ou bien vers la nécessité d'éliminer toutes les cellules cancéreuses - un objectif que les thérapies actuelles peinent à atteindre - ou bien vers l'introduction de nouvelles stratégies thérapeutiques qui cibleraient la régulation systémique de la propriété souche.

\section{L'identité souche est-elle stable?}

L'identité de la propriété souche doit être explorée pour chaque population de cellules souches, normales ou cancéreuses. Par exemple, les expériences d'ablation des cellules souches ou d'induction de dommages par irradiation dans certains tissus épithéliaux comme la peau, le sein ou la muqueuse intestinale, montrent que les cellules engagées dans la différenciation peuvent acquérir la propriété souche pour réparer le tissu ou maintenir son homéostasie [2-5]. Les données sont aujourd'hui insuffisantes pour déterminer plus précisément s'il s'agit d'une identité relationnelle ou systémique. Quoiqu'il en soit, des conversions similaires de cellules cancéreuses non souches en CSC sont également décrites dans les cancers qui se développent 
dans ces tissus, suggérant un maintien de cette identité [16, 22, 23$]$. La fréquence des dédifférenciations pourrait toutefois augmenter au cours de la progression dans certains cancers [24] et varier en fonction des types de cancers [25, 26]. Dans les cancers épidermoïdes, cette variabilité dépend de la cellule d'origine. La transition épithéliomésenchymateuse (TEM), au cours de laquelle les cellules cancéreuses peuvent se dédifférencier en CSC, est plus fréquente lorsque la cellule d'origine est une cellule du follicule pileux que lorsqu'il s'agit d'une cellule de l'épiderme interfolliculaire [25]. La TEM, qui serait induite par le microenvironnement au cours du développement normal du follicule pileux, pourrait devenir indépendante du microenvironnement dans les carcinomes épidermoïdes [27], suggérant une transition d'une identité relationnelle de la propriété souche vers une identité systémique.

Dans le système hématopoïétique, les connaissances actuelles suggèrent que la propriété souche est une propriété dispositionnelle. Elle ne semble pas pouvoir être acquise par des cellules normales engagées dans la différenciation et le microenvironnement joue un rôle déterminant dans le contrôle de l'activité des cellules souches (quiescence ou division, auto-renouvellement et/ou différenciation). Certains désordres génétiques et épigénétiques acquis lors de la transformation leucémique ou de la progression d'une hémopathie maligne pourraient remettre en cause l'identité dispositionnelle dans certaines hémopathies malignes ainsi que son maintien au cours de la progression de la maladie.

\section{Transition vers une identité catégorique?}

Certaines mutations oncogéniques provoquent l'activation anormale de voies de signalisation intracellulaires. Par exemple, dans les cellules hématopoïétiques, la mutation d'un gène codant une des protéines de la voie Ras (une situation observée dans la leucémie myélomonocytaire juvénile) induit une hyper-sensibilité des cellules souches et progénitrices hématopoïétiques à une cytokine, le granulocyte macrophage-colony stimulating factor (GM-CSF), présente dans leur microenvironnement [28]. La mutation JAK2 ${ }^{\mathrm{V} 617 \mathrm{~F}}$ observée dans certains néoplasmes myéloprolifératifs provoque aussi une hypersensibilité des cellules souches et progénitrices hématopoïétiques à des cytokines, dans ce cas l'érythropoïétine ou la thrombopoïétine? Ces altérations donnent-elles aux cellules souches leucémiques une indépendance vis-à-vis des signaux envoyés par leur niche? Toutes ces altérations induisent une phosphorylation activatrice de la protéine STAT5 (signal transducer and activator of transcription 5) dont le niveau d'activité dans les cellules souches hématopoïétiques régule la balance entre auto-renouvellement et différenciation. L'activation anormale de STAT5 pourrait perturber la régulation des cellules souches hématopoïétiques par leur environnement [29]. Les cellules hématopoïétiques portant la mutation JAK2 ${ }^{\mathrm{V} 617 \mathrm{~F}}$ sécrètent aussi une cytokine qui induit la dégradation de la niche hématopoïétique (elle provoque l'apoptose de cellules mésenchymateuses et de cellules de

${ }^{2}$ La mutation JAK2 ${ }^{\mathrm{V} 617 \mathrm{~F}}$ correspond à une substitution d'une guanine pour une thymine sur le gène JAK2 (Janus kinase 2) au codon 617, changeant une valine en une phénylalanine dans la protéine.
Schwann). Cette dégradation de la niche augmente la compétitivité des cellules malades par rapport aux cellules saines du fait d'une moindre dépendance des cellules mutées vis-à-vis de la niche $[30,31]$. Ces données questionnent le maintien de l'identité dispositionnelle dans les cellules souches leucémiques et suggèrent une transition vers une identité catégorique.

\section{Transition vers une identité systémique?}

Les altérations épigénétiques qui s'accumulent dans les cellules cancéreuses peuvent modifier l'identité de ces cellules, notamment en réactivant l'expression de gènes associés à la propriété souche. Par exemple, dans la leucémie lymphoïde chronique, l'accumulation stochastique de désordres locaux de la méthylation de I'ADN permet la ré-expression de gènes associés à la propriété souche dans des cellules non-souches, ce qui contribue à la progression de la maladie [32]. La réactivation épigénétique de propriétés associées aux cellules souches suggère la transition d'une identité catégorique vers une identité systémique dont on a vu qu'elle est actuellement une impasse thérapeutique. Des agents déméthylants sont couramment utilisés dans le traitement de certaines hémopathies myéloïdes aiguës ou chroniques, notamment chez les personnes âgées. La déméthylation induite par ces médicaments [33], qui n'induisent que des réponses transitoires, permet-elle la ré-expression de gènes associés à la propriété souche, compromettant toute réponse définitive en favorisant une transition identitaire?

\section{Transition vers une identité relationnelle?}

Chez les bovins, un parasite intracellulaire, Theileria annulata, transforme des cellules de la lignée monocytaire en induisant leur dédifférenciation (perte de marqueurs et de fonctions) $[34,35]$. Cette observation soulève deux questions. Cette transformation induitelle la propriété souche dans des cellules non-souches? Les données actuelles ne permettent pas de répondre à cette question mais la transformation cellulaire induite par le parasite, caractérisée par l'immortalisation des cellules infectées, provoque une forte reprogrammation de leurs voies de signalisation [36] $(\rightarrow)$. Si la transformation induit la propriété souche dans des cellules non- $(\rightarrow)$ Voir la Synthèse de K. Cheeseman et al., $m / s n^{\circ} 10$, octobre 2016, page 867 souches, quelle est alors l'identité de la propriété souche? L'identité souche supposée des cellules transformées est maintenue par des altérations épigénétiques induites par le parasite et réversibles avec son élimination, suggérant une identité dispositionnelle ou relationnelle en fonction du mécanisme de transmission 
du parasite. Si le parasite est transmis de manière verticale lors de la division des cellules infectées, l'identité souche est stable et donc dispositionnelle. Dans ce cas, le parasite a le même effet qu'un événement génétique (par exemple un réarrangement chromosomique générant une protéine de fusion $\mathrm{MOZ}-\mathrm{TIF}^{3}$ ou $\mathrm{MLL}^{3}-\varepsilon N L^{4}$ ) qui, lorsqu'il survient dans un progéniteur myéloïde engagé dans une voie de différenciation, le transforme en cellule propageant la leucémie [37, $38]^{5}$. En revanche, si la transmission du parasite est horizontale, de nouvelles cellules souches transformées peuvent être générées en permanence à partir de cellules non-souches et l'identité de la propriété souche est devenue relationnelle.

\section{Conclusion}

Il existe une grande diversité de cellules souches. Cette diversité concerne leurs attributs phénotypiques et fonctionnels mais aussi et plus fondamentalement, l'identité de leur propriété souche qui peut être catégorique, dispositionnelle, relationnelle, ou systémique en fonction de la capacité de cellules non-souches à acquérir la propriété souche et du rôle du microenvironnement. La distinction entre ces quatre identités est importante dans le contexte du cancer car elle impacte la stratégie thérapeutique. Le ciblage des CSC et celui de la niche reposent sur des présupposés distincts et leur efficacité dépend de l'identité de la propriété souche. L'accumulation d'altérations génétiques et épigénétiques au cours de la progression tumorale peut induire la transition d'une identité de la propriété souche vers une autre et cette transition pourrait justifier une adaptation thérapeutique. Les hémopathies malignes illustrent cette question : si l'identité de la propriété souche est dispositionnelle dans le système hématopoïétique, cette propriété semble pouvoir adopter l'une ou l'autre des identités décrites en situation pathologique. $\diamond$

\section{SUMMARY}

The identity of normal and cancer stem cells

What is a stem cell? Is stemness an intrinsic or extrinsic property? What role does the microenvironment play in the stemness identity? We distinguish four identities for normal and cancerous stem cells and explore their consequences for therapeutic strategy choice in the oncology setting. Acquisition of genetic and epigenetic alterations during cell transformation and disease progression questions the stability of the stemness property's identity in cancers. $\diamond$

\section{LIENS D'INTÉRÊT}

Les auteurs déclarent n'avoir aucun lien d'intérêt concernant les données publiées dans cet article.

\footnotetext{
${ }^{3}$ Fusion entre le gène codant $\mathrm{MOZ}$ (monocytic leukemia zinc finger protein), une histone acetyltransferase, et celui codant TIF2 (transcriptional intermediary factor 2), un co-activateur de transcription. ${ }^{4}$ Fusion entre les gènes codant MLL (mixed-lineage leukemia) et ENL (eleven nineteen leukemia).

${ }^{5}$ Le fait que ces cellules acquièrent des caractéristiques souches au cours de leur transformation peut susciter une interrogation concernant une éventuelle transition d'une identité dispositionnelle vers une identité relationnelle ou systémique. II est important de ne pas confondre changement d'identité cellulaire (une cellule non-souche qui devient souche) et changement d'identité de la propriété souche (la propriété souche passe d'une identité dispositionnelle à une identité relationnelle, par exemple). Dans le cas des translocations la cellule touchée change d'identité mais la propriété souche reste une propriété dispositionnelle.
}

\section{RÉFÉRENCES}

1. Gaillochet C, Lohmann JU. The never-ending story: from pluripotency to plant developmental plasticity. Development $2015 ; 142$ : 2237-49.

2. Blanpain C, Fuchs $\varepsilon$. Stem cell plasticity. Plasticity of epithelial stem cells in tissue regeneration. Science $2014 ; 344: 1242281$

3. Donati G, Watt FM. Stem cell heterogeneity and plasticity in epithelia. Cell Stem Cell $2015 ; 16: 465-76$.

4. Visvader JE, Clevers H. Tissue-specific designs of stem cell hierarchies. Nat Cell Biol 2016; $18: 349-55$.

5. Tetteh PW, Farin HF, Clevers H. Plasticity within stem cell hierarchies in mammalian epithelia. Trends Cell Biol $2015 ; 25: 100-8$.

6. Moyret-Lalle C, Pommier R, Bouard C, et al. Plasticité des cellules cancéreuses et dissémination métastatique. Med Sci (Paris) 2016 ; 32 : 725-31.

7. Schofield R. The relationship between the spleen colony-forming cell and the haemopoietic stem cell. Blood Cells $1978 ; 4: 7-25$.

8. Scadden DT. Nice neighborhood: Emerging concepts of the stem cell niche. Cell $2014 ; 157: 41-50$

9. Jagut M, Huynh J-R. Régulation des cellules souches de la lignée germinale. Med Sci (Paris) $2007 ; 23: 611-8$.

10. Barroca V, Lassalle B, Allemand I, et al. Des progéniteurs transplantés peuvent générer des cellules souches germinales. Med Sci (Paris) 2009 ; 25 893-5.

11. Adler CE, Sánchez Alvarado A. Types or states? Cellular dynamics and regenerative potential. Trends Cell Biol. $2015 ; 25$ : 687-96.

12. Zipori D. À la recherche d'une définition moléculaire plus que descriptive pour les cellules souches. Med Sci (Paris) 2011 ; 27 : 303-7.

13. Blau HM, Brazelton TR, Weimann JM. The evolving concept of a stem cell: entity or function? Cell $2001 ; 105: 829-41$.

14. Mikkers H, Frisen J. Deconstructing stemness. J Eur Mol Biol Organ 2005 ; 24 2715-9.

15. Laplane L. Cancer stem cells: Philosophy and therapies. Cambridge, MA : Harvard University Press, 2016.

16. Gupta PB, Fillmore CM, Jiang G, et al. Stochastic state transitions give rise to phenotypic equilibrium in populations of cancer cells. Cell $2011 ; 146: 633-44$

17. Kreso A, Dick JE. Evolution of the cancer stem cell model. Cell Stem Cell $2014 ; 14: 275-91$.

18. Reya T, Morrison SJ, Clarke MF, et al. Stem cells, cancer, and cancer stem cells. Nature $2001 ; 414$ : 105-11.

19. Häfner S, Coulombel L. L'oligarchie contestée des cellules souches cancéreuses. Med Sci (Paris) $2009 ; 25: 227-8$.

20. Reynaud D, Pietras $\varepsilon$, Barry-Holson K, et al. IL-6 controls leukemic multipotent progenitor cell fate and contributes to chronic myelogenous leukemia development. Cancer Cell 2011; 20 : 661-73.

21. Welner RS, Amabile G, Bararia D, et al. Treatment of chronic myelogenous leukemia by blocking cytokine alterations found in normal stem and progenitor cells. Cancer Cell $2015 ; 27: 671-81$.

22. Chaffer CL, Brueckmann I, Scheel C, et al. Normal and neoplastic nonstem cells can spontaneously convert to a stem-like state. Proc Natl Acad Sci USA $2011 ; 108: 7950-5$.

23. Vermeulen L, De Sousa EMF, van der Heijden M, et al. Wnt activity defines colon cancer stem cells and is regulated by the microenvironment. Nat Cell Biol $2010 ; 12: 468-76$.

24. Thirant $C$, Bessette $B$, Varlet $P$, et al. Clinical relevance of tumor cells with stem-like properties in pediatric brain tumors. PLoS One 2011; 6 : el6375.

25. Latil M, Nassar D, Beck B, et al. Cell-type-specific chromatin states differentially prime squamous cell carcinoma tumor-initiating cells for epithelial to mesenchymal transition. Cell Stem Cell 2017 ; 20 : 191-204.e5.

26. Chaffer CL, Marjanovic ND, Lee T, et al. Poised chromatin at the ZEBI promoter enables breast cancer cell plasticity and enhances tumorigenicity. Cell $2013 ; 154: 61-74$.

27. Whitson RJ, Oro AE. Soil primes the seed: Epigenetic landscape drives tumor behavior. Cell Stem Cell 2017 ; 20 : 149-50.

28. Emanuel PD, Bates LJ, Castleberry RP, et al. Selective hypersensitivity to granulocyte-macrophage colony-stimulating factor by juvenile chronic myeloid leukemia hematopoietic progenitors. Blood $1991 ; 77$ : 925-9.

29. Staerk J, Constantinescu SN. The JAK-STAT pathway and hematopoietic stem cells from the JAK2 V617F perspective. JAK-STAT 2012 ; 1 : 184-190.

30. Vainchenker W, Constantinescu SN, Plo I, et al. Recent advances in understanding myelofibrosis and essential thrombocythemia. F1000Research $2016 ; 5: 700$. 


\section{RÉFÉRENCES}

31. Arranz L, Sánchez-Aguilera A, Martín-Pérez D, et al. Neuropathy of haematopoietic stem cell niche is essential for myeloproliferative neoplasms. Nature $2014 ; 512: 78-81$.

32. Landau DA, Clement K, Ziller MJ, et al. Locally disordered methylation forms the basis of intratumor methylome variation in chronic lymphocytic leukemia. Cancer Cell $2014 ; 26: 813-25$

33. Merlevede J, Droin N, Qin T, et al. Mutation allele burden remains unchanged in chronic myelomonocytic leukaemia responding to hypomethylating agents. Nat Commun $2016 ; 7$ : 10767.

34. Sager H, Davis WC, Dobbelaere DA, et al. Macrophage-parasite relationship in theileriosis. Reversible phenotypic and functional dedifferentiation of macrophages infected with Theileria annulata. J Leukoc Biol 1997 ; $61: 459-68$.

35. Sager $H$, Bertoni $G$, Jungi TW. Differences between $B$ cell and macrophage transformation by the bovine parasite, Theileria annulata: a clonal approach. J Immunol 1998 ; 161 : 335-41.
36. Cheeseman K, Certad G, Weitzman JB. Parasites et cancer : existe-t-il un lien? Med Sci (Paris) $2016 ; 32: 867-73$.

37. Huntly BJ, Shigematsu H, Deguchi K, et al. MOZ-TIF2, but not BCR$A B L$, confers properties of leukemic stem cells to committed murine hematopoietic progenitors. Cancer Cell $2004 ; 6$ : 587-96.

38. Cozzio A, Passegue $\varepsilon$, Ayton PM, et al. Similar MLL-associated leukemias arising from self-renewing stem cells and short-lived myeloid progenitors. Genes Dev 2003 ; 17 : 3029-35

\section{TIRÉS À PART}

L. Laplane

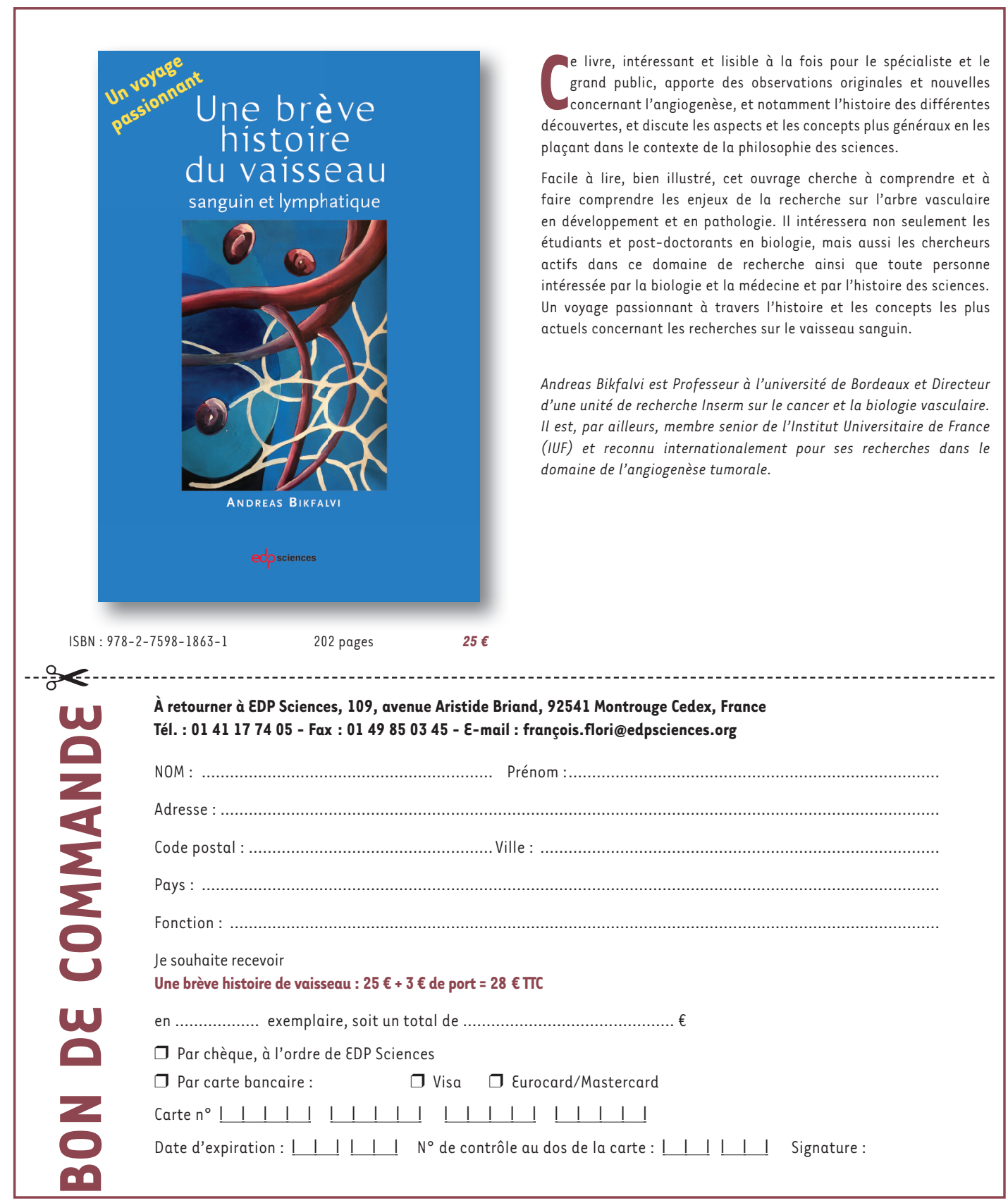

